\title{
Delayed administration of neural stem cells after hypoxia-ischemia reduces sensorimotor deficits, cerebral lesion size, and neuroinflammation in neonatal mice
}

\author{
Luca Braccioli ${ }^{1,2}$, Cobi J. Heijnen ${ }^{3}$, Paul J. Coffer ${ }^{2}$ and Cora H. Nijboer ${ }^{1}$
}

\begin{abstract}
BACKGROUND: Hypoxic-ischemic (HI) encephalopathy causes mortality and severe morbidity in neonates. Treatments with a therapeutic window $>6 \mathrm{~h}$ are currently not available. Here, we explored whether delayed transplantation of allogenic neural stem cells (NSCs) at $10 \mathrm{~d}$ after HI could be a tool to repair HI brain injury and improve behavioral impairments. METHODS: HI was induced in 9-d-old mice. Animals received NSCs or vehicle intracranially in the hippocampus at $10 \mathrm{~d}$ post$\mathrm{HI}$. Sensorimotor performance was assessed by cylinder rearing test. Lesion size, synaptic integrity, and fate of injected NSCs were determined by immuno-stainings. Neuroinflammation was studied by immuno-stainings of brain sections, primary glial cultures, and TNFa ELISA.
\end{abstract}

RESULTS: NSC transplantation at $10 \mathrm{~d}$ post-insult induced long-term improvement of motor performance and synaptic integrity, and reduced lesion size compared to vehicletreatment. HI-induced neuroinflammation was reduced after NSC treatment, at least partially by factors secreted by NSCS. Injected NSCs migrated toward and localized at the damaged hippocampus. Transplanted NSCs differentiated toward the neuronal lineage and formed a niche with endogenous precursors.

CONCLUSION: Our study provides evidence of the efficacy of NSC transplantation late after $\mathrm{HI}$ as a tool to reduce neonatal HI brain injury through regeneration of the lesion.

N eural stem cells (NSCs) are pluripotent cells that can selfrenew and differentiate into neurons, astrocytes, and oligodendrocytes (1). NSCs can be found both in the developing embryo during neurogenesis identified as radial glia, and in specialized niches of the adult brain such as the subventricular zone (SVZ) and the granular layer of the dentate gyrus (DG) of the hippocampus (2). NSCs can be isolated from the developing cortex or from the adult niches and expanded in vitro as neurospheres (3). Several studies using animal models of neurodegenerative pathologies show that NSC transplantation is a promising tool for treating conditions affecting the central nervous system (4). NSC transplantation has been shown to improve cognition in a transgenic mouse model of Alzheimer's disease (5), to induce recovery in a murine model of multiple sclerosis (6), to improve motor function in mice with Machado-Joseph disease (7), and to promote recovery after neonatal excitotoxic brain injury (8).

Hypoxia-ischemia (HI) during the perinatal period is a severe condition with high mortality and major long-term neurodevelopmental consequences for the survivors $(9,10)$. Affected neonates often suffer from brain damage as a major consequence of $\mathrm{HI}$ leading to long-term sequelae including cerebral palsy, mental retardation, seizures, and behavioral and psychological problems.

After a HI insult, NSCs expand within the subventricular zone (SVZ) to participate in an endogenous regenerative response $(11,12)$. However, this endogenous restorative capacity is limited due to HI-induced apoptosis of NSC-derived neural precursors hampering the generation of new functional neurons and glial cells (13). To circumvent these limitations and improve the endogenous regenerative potential of the neonatal brain after HI, a number of preclinical studies by our group and others aimed at either replacing the endogenous stem cells or boost their differentiation capacity, for instance by intracranial delivery of NSCs or intranasal application of mesenchymal stem cells (14-16).

Current treatment options for neonatal HI brain injury are scarce and are mostly aimed at reducing early brain damage (e.g., by whole body hypothermia) and therefore rely on a strictly timed application within a few hours $(<6 \mathrm{~h})$ after the insult $(17,18)$. Therefore, there is a need for new efficacious regenerative strategies like stem cell transplantation that can be applied with a wider therapeutic time-window than the established interventions at present.

A few recent preclinical studies have shown how NSC transplantation ameliorates functional impairment and reduces tissue damage after neonatal HI. However, these studies only reported administration of NSCs within 1-3 d after HI $(14,19,20)$. The aim of the present study is to investigate

\footnotetext{
'Laboratory of Neuroimmunology and Developmental Origins of Disease (NIDOD), University Medical Center Utrecht, Utrecht, The Netherlands; ${ }^{2}$ Center for Molecular Medicine

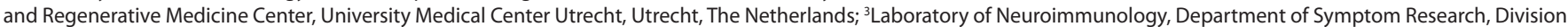
of Internal Medicine, University of Texas MD Anderson Cancer Center, Houston, Texas. Correspondence: Luca Braccioli (I.braccioli@umcutrecht.nl)

Received 24 February 2016; accepted 7 July 2016; advance online publication 5 October 2016. doi:10.1038/pr.2016.172
} 


\section{Articles | Braccioili tal.}

whether late administration of NSCs (at $10 \mathrm{~d}$ after insult) has a beneficial effect on HI-induced deficits in motor behavior, lesion size, and neuroinflammation. We also aimed to elucidate the engraftment and fate of transplanted NSCs.

\section{RESULTS}

\section{Intracranial NSC Treatment at $10 \mathrm{~d}$ After HI Improves Motor Performance}

P9 mouse pups underwent $\mathrm{HI}$ and were treated with an intracranial injection of 100,000 allogenic NSCs or vehicle solution at $10 \mathrm{~d}$ post-insult (for experimental set-up see Figure 1a). By using the cylinder rearing test (CRT), we investigated whether delayed NSCs transplantation could restore deficits in motor behavior induced by HI. At 10, 28, and $56 \mathrm{~d}$ after the HI insult, HI-VEH animals showed a significant preference to use the nonimpaired forepaw when compared to sham-operated littermates (SHAM) indicating HI-induced motor impairment (Figure 1b). NSC-treated animals showed a strong reduction in nonimpaired forepaw preference at both 28 and $56 \mathrm{~d}$ post-HI when compared to HI-VEH mice (Figure $\mathbf{1 b}$ ). The reduction in motor impairment after NSC treatment remained steady between 28 and $56 \mathrm{~d}$ after the insult indicating longterm improvement of motor behavior. In order to assure that the HI-VEH and HI-NSC experimental groups had a comparable degree of motor impairment upon $\mathrm{HI}$, mice were tested in the CRT prior to treatment ( $10 \mathrm{~d}$ after $\mathrm{HI}$ ) before randomly dividing the pups among the different groups. Figure $\mathbf{1 b}$ shows

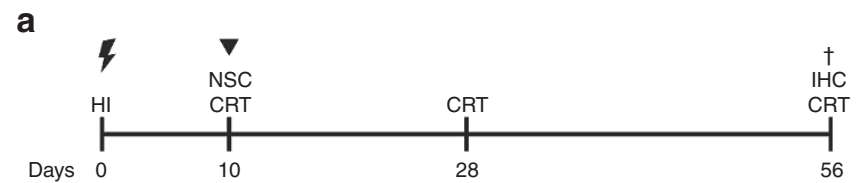

b

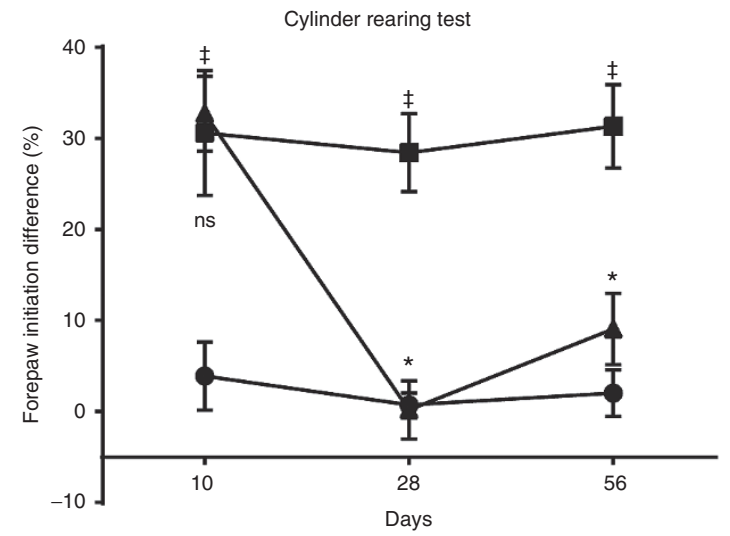

Figure 1. Effect of neural stem cells (NSC) treatment on sensorimotor behavior over time. (a) Experimental set-up showing day of hypoxicischemic (HI) surgery or sham operation (lightning), intracranial NSC or vehicle treatment (arrowhead) and termination at day 56 after $\mathrm{HI}$ (cross). Timepoints of cylinder rearing test (CRT) are shown over time (i.e., 10, 28 , and $56 \mathrm{~d}$ after $\mathrm{HI}$ ) (b) CRT results show that NSC treatment improves motor performance at 28 and $56 \mathrm{~d}$ after $\mathrm{HI}$ as compared to vehicle treatment. CRT at $10 \mathrm{~d}$ post-HI is performed prior to treatment. Circles: SHAM; squares: VEH; triangles: NSC. Animal numbers: $10 \mathrm{~d}$ SHAM: $n=8$, VEH: $n=7$, NSC: $n=9.28$ d SHAM: $n=9$, VEH: $n=9$, NSC: $n=7.56$ d SHAM: $n=9$, VEH: $n=6$, NSC: $n=9$. $¥ P<0.001$ : VEH vs. sham-controls; ${ }^{*} P<0.001$ : NSC vs. VEH; ns = not significant with two-way ANOVA and LSD post-test. that $\mathrm{HI}$-induced preference to use the nonimpaired forepaw was similar in VEH- and NSC-treated mouse pups prior to treatment (i.e., day 10).

Intracranial NSC Treatment Reduces Infarct Size and Preserves Synaptic Integrity in the Ipsilateral Hemisphere

To assess the long-term effect of NSC treatment on lesion size and specific neuronal and white matter damage, mice were sacrificed at $56 \mathrm{~d}$ post-HI (Figure 1a). Brains were stained for hematoxylin eosin $(\mathrm{HE})$ to assess ipsilateral volume loss, for microtubule-associated protein 2 (MAP2) to assess ipsilateral neuronal loss, for synaptophysin to assess synaptic integrity. Analysis of HE staining showed that $\mathrm{HI}$ induced $18 \pm 3 \%$ loss of ipsilateral area in VEH-treated animals with a clear lesion in the hippocampal area and some loss of volume in the cortex and thalamic regions (Figure 2a,b). Importantly, NSC treatment significantly reduced volume loss to only $9 \pm 4 \%$ loss in the ipsilateral hemisphere when compared to VEH (Figure 2a,b). Sham-operated littermates did not show any area loss.

In addition, Figure $2 \mathrm{c}$ shows that HI-induced ipsilateral neuronal damage was significantly reduced after NSC treatment (13 $\pm 8 \%$ MAP2 loss) when compared to VEH-treated animals ( $20 \pm 6 \%$ MAP2 loss). No MAP2 loss was observed in sham-operated animals (Figure 2c,d). MAP2 loss in HI animals was primarily observed in the hippocampal area.

As is shown in Figure 2e,f, synaptophysin-positive vesicles (dark-brown dots, see arrows) were observed throughout the hippocampus in sham-operated animals (arrows), indicating intact functional neurons. HI induced massive loss of synaptophysin staining both in the CA3 and DG areas of the hippocampus respectively (number signs) (Figure 2e,f). In some vehicle-treated animals, the hippocampus was severely infarcted with complete loss of synaptophysin staining (data not shown). NSC-treated animals showed the presence of synaptophysin-positive vesicles (arrows) in the CA3 and DG. These data indicate that upon HI, NSCs might prevent the loss of previously formed synapses or might be involved in formation of new synapses during repair of the damaged areas (Figure 2e,f).

\section{Localization and Fate of Transplanted NSCs}

In order to investigate where NSCs home to in the HI-damaged brain after intracranial transplantation, NSC were labeled with PKH26 and injected intracranially into mice at $10 \mathrm{~d}$ post-HI (for experimental set-up, see Figure 3a). Animals were sacrificed $3 \mathrm{~d}$ after transplantation and brains were sectioned at the hippocampal level as the injection site was within the damaged ipsilateral hippocampus (number sign in Figure 3b). At $3 \mathrm{~d}$ after injection, PKH26 signal was detectable in (i) multiple areas of the damaged ipsilateral hippocampus aligning the ipsilateral dentate gyrus (DG) (red boxes, Figure 3b), (ii) the area of the dorsal third ventricle (blue boxes, Figure $3 \mathbf{b}$ ), and (iii) the third ventricle (green box, Figure 3b).

In order to determine the fate of the PKH26+ NSCs, immunofluorescent analyses for progenitor- and lineagespecific markers were performed. At $3 \mathrm{~d}$ after transplantation, 
a

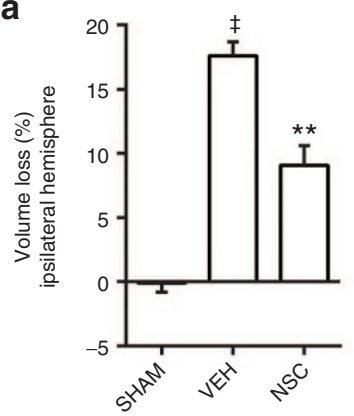

b

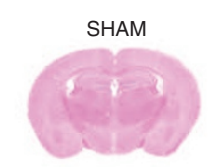

VEH

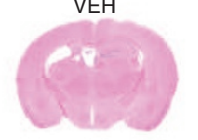

NSC

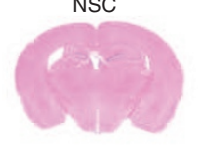

e

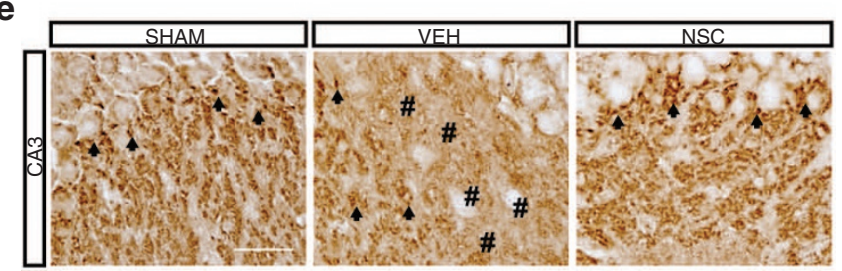

f

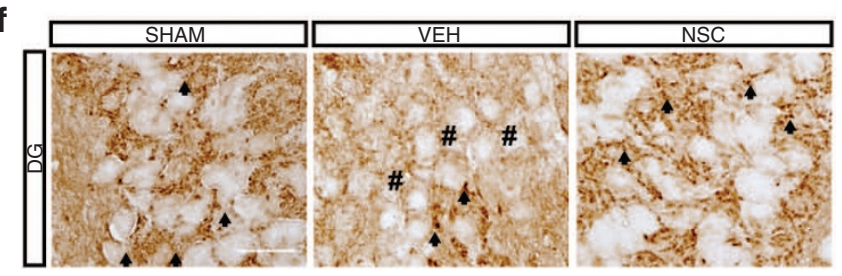

Figure 2. Effect of neural stem cells (NSC) treatment on lesion volume, gray matter injury and synapse loss at $56 \mathrm{~d}$ after hypoxic-ischemic ( $\mathrm{HI})$. (a) NSC treatment significantly reduces Hl-induced volume loss in the ipsilateral hemisphere. (b) Representative sections of HE staining in all experimental groups. (c) NSC treatment significantly reduced $\mathrm{HI}$-induced MAP2 loss in the ipsilateral hemisphere. (d) Representative sections of MAP2 staining in all experimental groups. (e-f) Representative sections of synaptophysin staining at CA3 (e) and DG (f) areas of the hippocampus. Synaptophysin-positive vesicles are shown at arrowheads. NSC treatment reduces loss of synaptophysin staining as observed in vehicle-treated littermates (number signs). Scale bar $30 \mu \mathrm{m}$. $\neq P<0.001$ : VEH vs. shamcontrol; ${ }^{*} P<0.05$, ${ }^{*} P<0.001$ : NSC vs. VEH with one-way ANOVA and LSD post-test. Animal numbers: HE SHAM: $n=9, \mathrm{VEH}: n=9, \mathrm{NSC}: n=9$. MAP2 SHAM: $n=9$, VEH: $n=9$, NSC: $n=9$.

PKH $26^{+}$cells are not expressing the stem cell marker Nestin (Figure 3c,d). However PHK26 cells were found in proximity of Nestin ${ }^{+}$PHK26- cells (Figure 3c,d) suggesting that the injected exogenous NSCs might interact with endogenous progenitors in the brain as previously reported (4). Staining for the neuroblast marker Doublecortin (DCX) revealed the presence of double-positive $\mathrm{PKH}_{26} 6^{+} / \mathrm{DCX}^{+}$cells (Figure $3 \mathrm{c}$,d) indicating that at least a part of the injected NSCs had differentiated towards young migrating neuroblasts at $3 \mathrm{~d}$ postinjection. Conversely, Glial fibrillary acidic protein (GFAP) astrocytes could also be found surrounding the areas of $\mathrm{PKH} 26$ signal, but no cellular colocalization of GFAP and PKH26 signal could be identified (Figure 3c,d). These data indicate that the injected NSCs are not differentiating towards the astrocyte lineage.

\section{Migration of NSCs Toward the Lesion Site After Transplantation in the Contralateral Hippocampus}

In order to investigate the migratory capacity of transplanted NSCs toward the damaged areas, we injected NSCs in the contralateral, undamaged hippocampus (Figure 4). At $5 \mathrm{~d}$ after transplantation, the NSCs were detected within the DG of the ipsilateral hippocampus and in other hippocampal regions surrounding the lesion (Figure 4). Strikingly, hardly any NSCs were detected in the contralateral hippocampus at the injection site, meaning that the majority of the transplanted cells migrated after transplantation, most likely through a chemoattractive gradient generated by the damaged milieu.

\section{Neuroinflammation in the HI-Damaged Brain Is Attenuated by NSC Transplantation}

We next determined whether NSC treatment could have an effect on the neuroinflammatory response as a consequence of the $\mathrm{HI}$ insult. We determined Iba- 1 and GFAP expression at $5 \mathrm{~d}$ after transplantation ( $15 \mathrm{~d}$ after $\mathrm{HI}$ ) as measures of microglia activation and astrogliosis respectively (see Figure 3, panel a for set-up). Figure 5a-d shows that HI induces a strong upregulation of Iba-1 and GFAP at $15 \mathrm{~d}$ after $\mathrm{HI}$ in vehicle-treated animals, especially in the hippocampal area. NSC treatment reduced the upregulation of the Iba-1+ and GFAP+ signal in this area almost completely at $5 \mathrm{~d}$ after transplantation (Figure 5a-d) indicating that NSC transplantation dampens the neuroinflammatory response. In order to investigate whether the observed dampening of neuroinflammation could be directly mediated by the NSCs secretome, primary microglia were cultured in presence of conditioned medium (CM) derived from cultured NSCs or control nonconditioned medium (CTR). Figure $5 \mathrm{e}$ shows that microglia cultured in $\mathrm{CM}$ produced significantly less TNFa, when compared to microglia cultured in CTR medium. Moreover, Ibal expression level per cell was markedly decreased after CM culture when compared to CTR (Figure 5f,g). As both TNFa production and Ibal expression levels are markers of microglial activation $(21,22)$, these data together indicate that reduced microglial activation can be a direct effect of the NSCs' secretome.

\section{DISCUSSION}

The present study shows the beneficial effect of late allogenic intracranial NSC transplantation for HI brain injury. We show that NSCs are remarkably potent in attenuating motor impairment in HI-injured mice with a stable and long-lasting effect. Moreover, the HI-induced ipsilateral volume loss and neuronal loss was reduced after late NSC treatment. We have shown 


\section{Articles | Braccioli et al.}

a

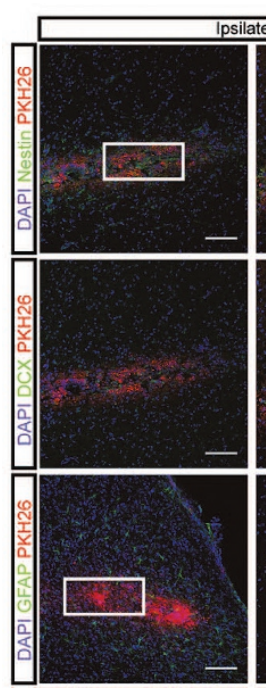

Days 0
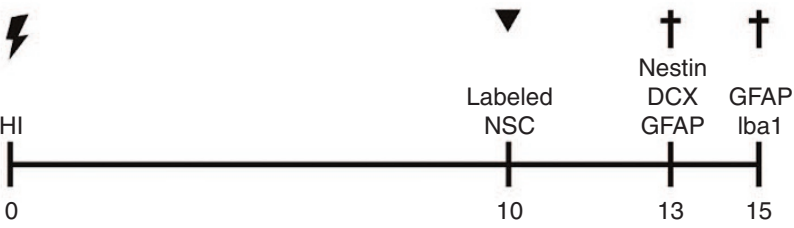

b

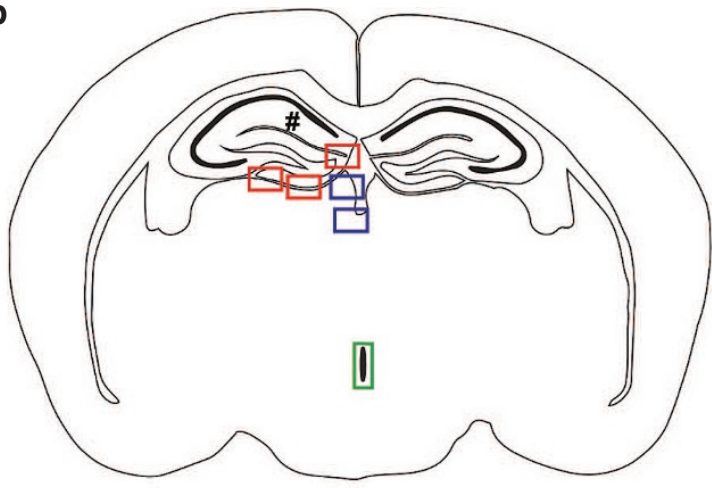

d
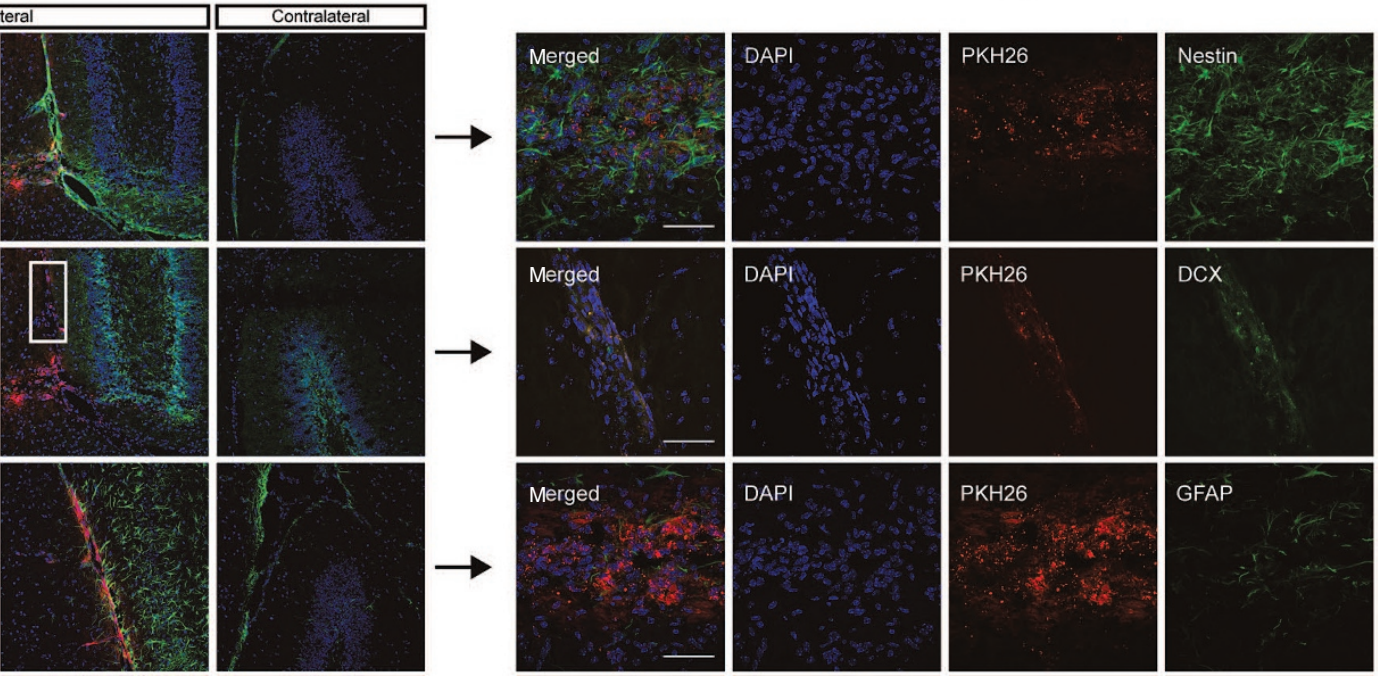

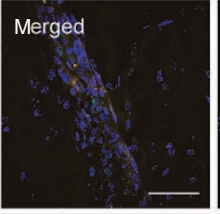

Merged
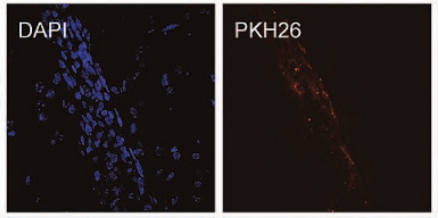

DAPI
PKH26

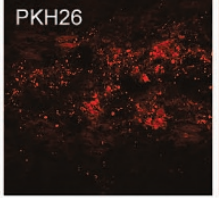

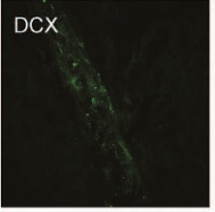

GFAP
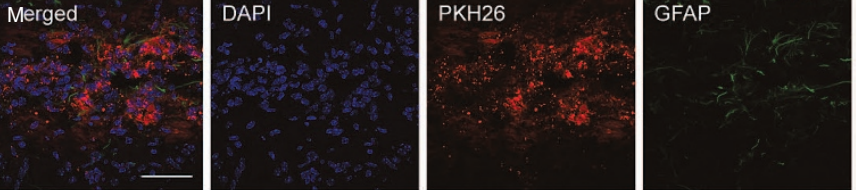

Figure 3. Localization and fate of injected neural stem cells (NSCs) in the hypoxic-ischemic (HI)-damaged brain. (a) Experimental set-up showing day of HI surgery or sham operation (lightning), intracranial PKH26-labeled NSC or vehicle treatment (arrowhead) and termination days (crosses; i.e., 13 and $15 \mathrm{~d}$ post-HI). (b) Coronal brain section at hippocampal level illustrating the injection site of NSCs (number sign) and showing the localization of PKH26 signal at the hippocampus (red boxes), dorsal third ventricle (blue boxes), and third ventricle (green box). (c) Representative sections of mice sacrificed $3 \mathrm{~d}$ after NSC treatment showing localization of PHK26 (red) signal at the ipsilateral hippocampus. Sections were stained for Nestin, DCX and GFAP (green) as indicated and nuclei counterstained with DAPI (blue). No PKH26 signal was detected in the contralateral hippocampus. Boxes indicate areas depicted in Figure 3d. Scale bar $100 \mu \mathrm{m}$. (d) Z-stack images showing colocalization (yellow) of PKH26 signal (red) with Nestin, DCX and GFAP (green) as indicated. Nuclei were counterstained with DAPI (blue). Scale bar $50 \mu \mathrm{m}$.

before that the HI-induced lesion in our model is already fully established at $3 \mathrm{~d}$ post-HI showing disintegration of the hippocampal architecture (23). This previous study showed that between day 3 and 15 after HI, there was no significant increase in lesion size indicating that the applied NSCs at day 10 after $\mathrm{HI}$ actually repaired the established lesion. Although ipsilateral hippocampal volume was not recovered fully, MAP2 staining showed that NSCs aided in repair and reconstruction of the hippocampal structure. Synaptic integrity in the ipsilateral hippocampus was maintained or repaired after transplantation of NSCs. We observed that transplanted NSCs had differentiated into DCX+ neuroblasts. These neuroblasts were found in proximity of endogenous astrocytes and progenitor cells indicating formation of a neurogenic niche in the lesion area. Interestingly, we showed that transplanted NSCs have the capacity to migrate to the damaged area when injected at a site more distant from the lesion. Additionally, the neuroinflammatory reaction evoked by $\mathrm{HI}$, as measured by microglia activation and gliosis in the area of the ipsilateral hippocampus, were strongly reduced by NSC treatment. Reduction of microglia activation could be directly mediated by the NSCs secretome, as we observed a reduction in TNFa production and Ibal expression by microglia when cultured in presence of NSC-conditioned medium.

There are a few previous studies that have explored the therapeutic potential of NSC transplantation in different ischemic models. Daadi et al. investigated the effect of intracranial human NSC transplantation at $24 \mathrm{~h}$ after $\mathrm{HI}$ in 7 -d-old rats (14). Consistent with our results, Daadi et al. showed an improvement in motor outcome in NSC-treated animals upon HI. Moreover, this study showed that transplanted human NSCs differentiated towards the neuronal as well as the astrocytic lineage (NSCs becoming GFAP-positive cells). These data are partially in contrast with our results as we showed that all the detected NSCs had differentiated towards young migrating neuroblasts (DCX-positive cells) and we did not detect any NSCs expressing the astrocyte marker GFAP. This could be explained by the difference in the timing of transplantation 
a

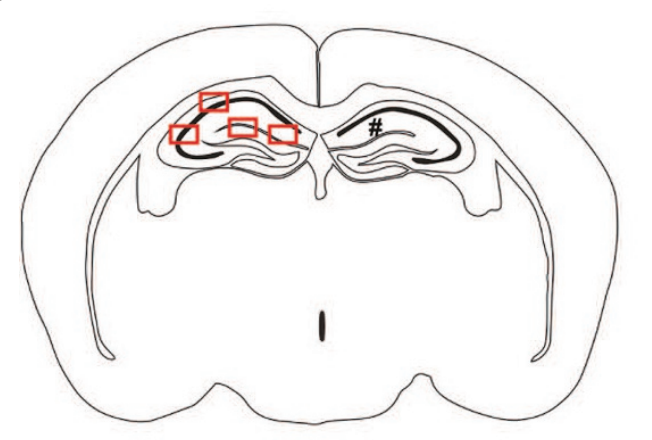

b

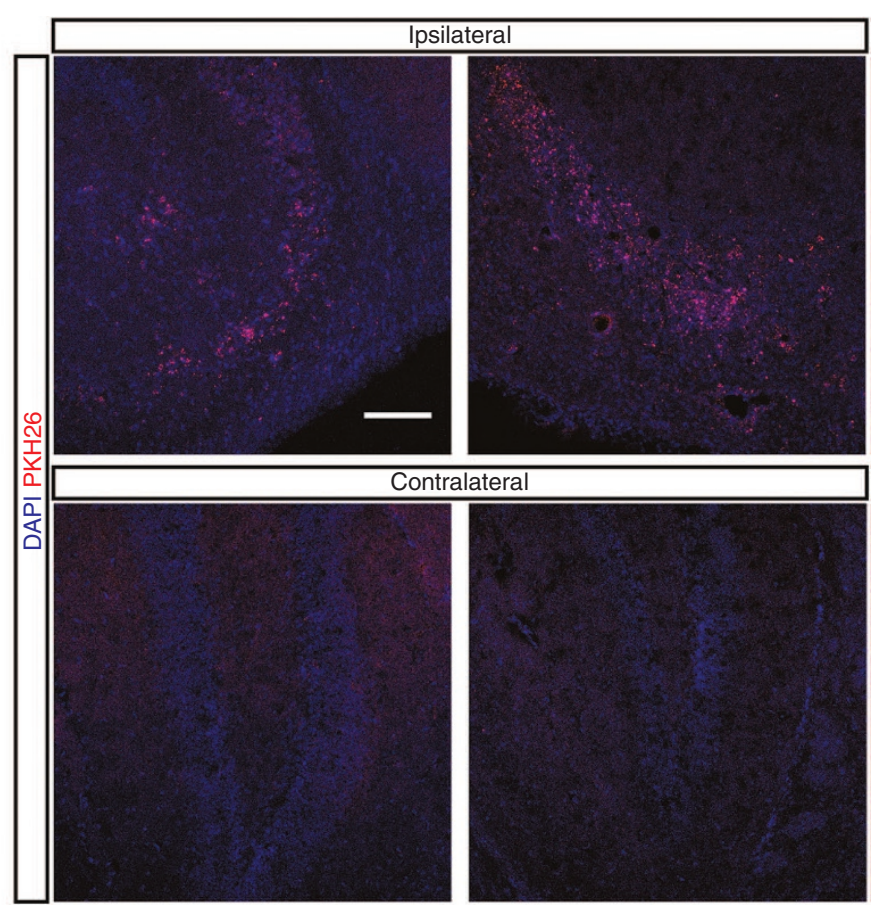

Figure 4. Neural stem cells (NSCs) migrate to the lesion site. (a) Coronal brain section at hippocampal level illustrating the injection site of NSCs (number sign) in the contralateral hippocampus and showing the localization of PKH26 signal in the ipsilateral hippocampus (red boxes). (b) Representative pictures of mice sacrificed $5 \mathrm{~d}$ after NSC treatment showing localization of PHK26 (red) signal in the ipsilateral hippocampus. Hardly any PKH26 signal was detected in the contralateral hippocampus. Nuclei were counterstained with DAPI (blue). Scale bar $100 \mu \mathrm{m}$.

between our study and the one from Daadi and his colleagues. In line with this explanation, Rosenblum et al. reported how early (6-24h) transplantation of NSCs in adult stroke mice led to increased differentiation of NSCs into astrocytes, however delayed (7-14 d) transplantation resulted in neuronal differentiation (24). Moreover, Titomanlio et al. show that NSCs implanted into the neonatal excitotoxic injured brain early after damage (4-72h) differentiate toward both neurons and oligodendrocytes, before undergoing apoptosis (8). These studies together indicate that the milieu of the damaged brain might change over time modulating the differentiation and survival capacity of the grafted NSCs and that it could be more beneficial to delay regenerative treatment of patients with HIE in order to improve selective neuronal repair.

Daadi et al. did not observe any effect on lesion size after NSC treatment while we observed a potent beneficial effect of late NSC treatment on gray matter loss. This difference could be explained by the source of the transplanted NSCs, as murine (although allogenic) NSCs used in our present study might engraft better than human NSCs in the murine host brain. This hypothesis could be supported by the fact that Daadi et al. observed an increase in Iba1-positive microglia in the transplanted area, whereas our data showed a reduction of Iba1-positive cells in the area surrounding the damaged hippocampus, indicating that the xenograft of human NSCs might elicit an inflammatory response in the brain.

Another study using neonatal HI mice showed the interaction of the host brain with mouse NSC grafts seeded onto a polymer scaffold, which was implanted at $7 \mathrm{~d}$ after $\mathrm{HI}$ in the infarction cavity (25). This study showed how the grafted NSCs differentiated into neurons and oligodendrocytes, resulting in long-distance neuronal projections. Consistent with our study, Park and colleagues showed that the neuroinflammatory response, i.e., astroglial scar formation and microglia infiltration, were minimized after NSC transplantation.

Previous studies have shown that transplanted NSCs do express mature neuronal markers within several days after transplantation $(14,25)$. Although we did not assess long-term fate of the transplanted NSCs, our histological and behavioral data indicate that NSC transplantation eventually led to long-term functional recovery and regeneration of the hippocampal structure. We suggest therefore that the transplanted NSCs indeed might have integrated as functional differentiated neurons. In line, our data on synaptic integrity showed a potent effect of NSC treatment on synapse formation in the hippocampal area. Increased synaptophysin expression after NSC treatment can be a result of preservation of existing synapses or induction of new synapses by newly-formed neurons from the transplant.

The transplanted NSCs were detected in damaged areas of the hippocampus when transplanted in the contralateral hemisphere, indicating that NSCs are capable of migrating towards damage signals. This is in line with previous studies showing that injected NSCs can respond to chemoattractants (like stromal cell-derived factor $1 \alpha($ SDF- $1 \alpha)$ ) and migrate toward injury sites (26). This finding is in favor of a possible clinical application of NSC transplantation as less invasive administration routes (e.g., intravenous, intranasal) might result in NSCs successfully reaching the lesion site. 


\section{Articles $\mid$ Braccioli et al.}

a

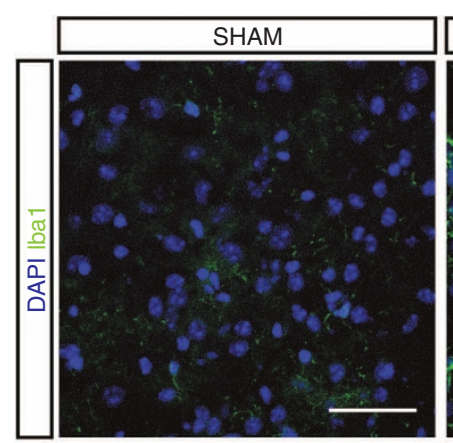

C

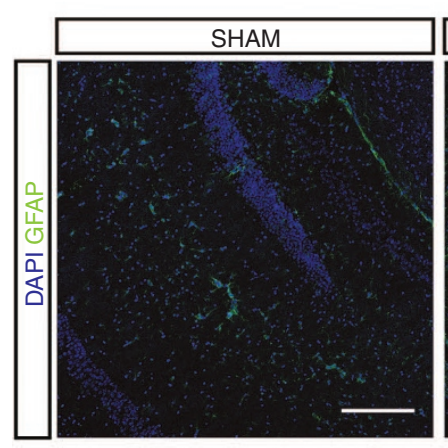

e

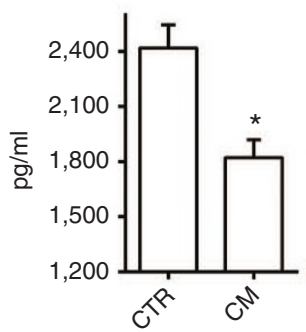

f
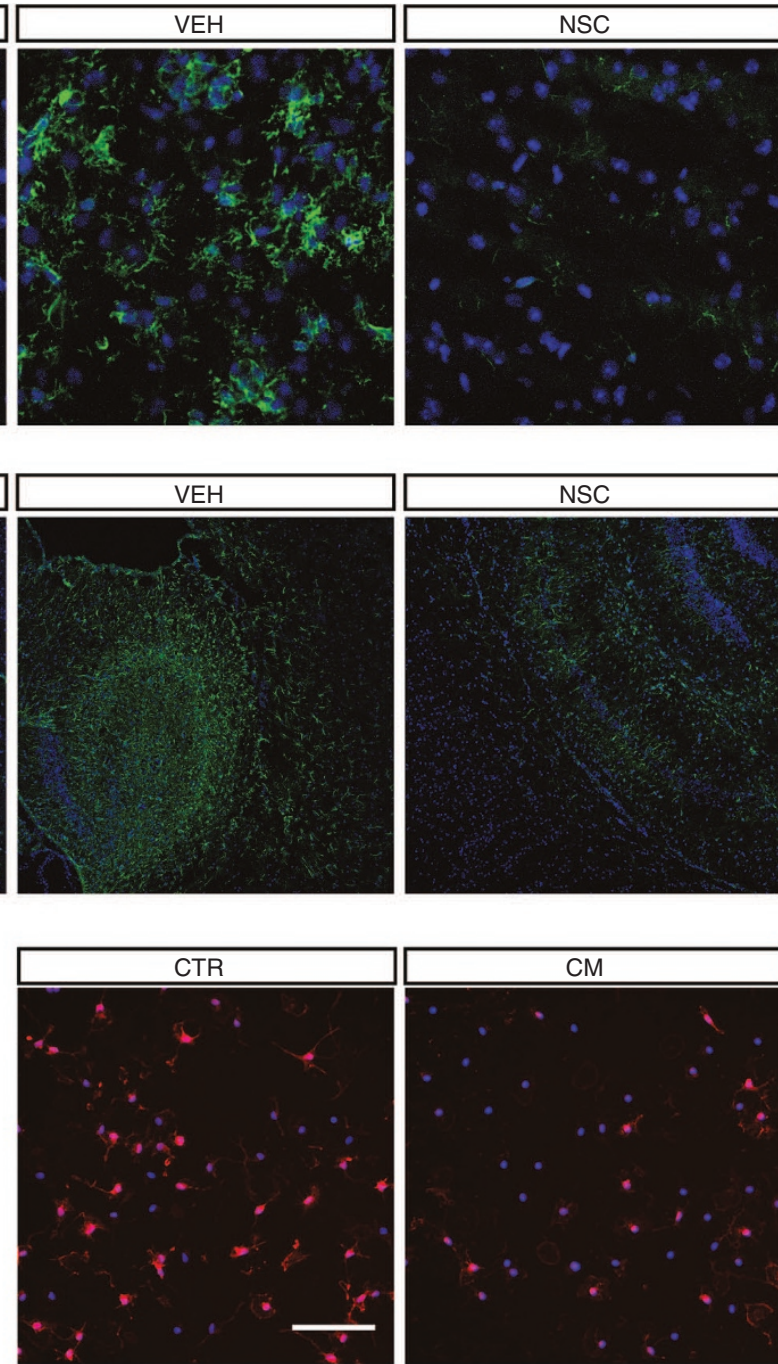
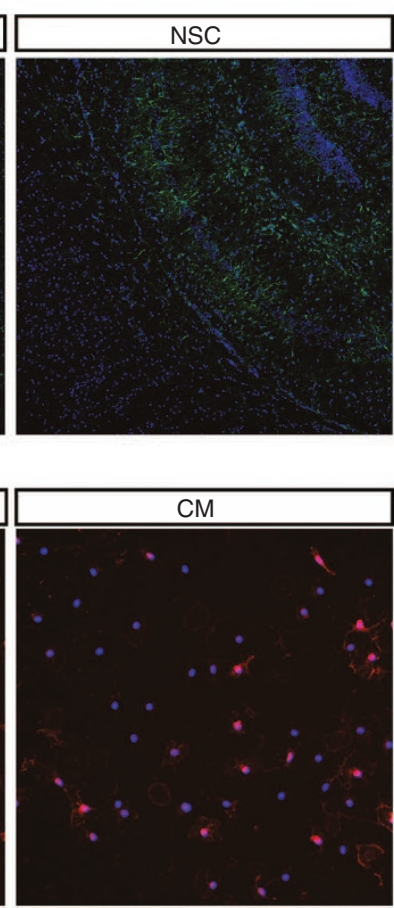

b

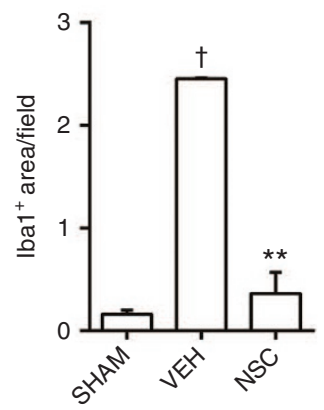

d

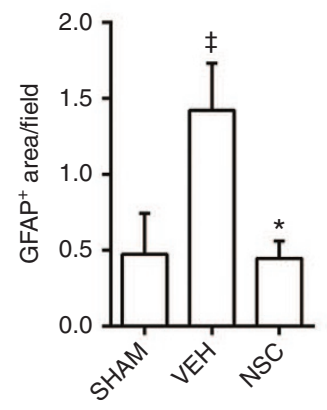

g

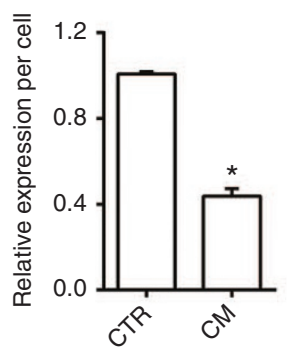

Figure 5. Effect of neural stem cells (NSC) treatment on neuroinflammation after hypoxic-ischemic (HI). For experimental set-up, see Figure 3a. (a) Representative sections showing microglia activation (Iba1 (green)) in the ipsilateral hippocampus. Scale bar $50 \mu m$. (b) Quantification of Iba-1-positive signal showing that NSC treatment significantly reduces microglia activation. (c) Representative sections showing GFAP (green) signal in the ipsilateral hippocampus. Scale bar $250 \mu \mathrm{m}$. (d) Quantification of GFAP-positive signal showing that NSC treatment significantly reduces astrocyte activation $\neq P<0.05,+P<0.005$ : HI-vehicle vs. sham-control; ${ }^{*} P<0.05,{ }^{* *} P<0.001$ : NSC treatment vs. vehicle treatment analyzed with one-way ANOVA, LSD post-test. Animal numbers: Iba1: SHAM: $n=2$, VEH: $n=3$, NSC: $n=5$. GFAP SHAM: $n=2$, VEH: $n=3$, NSC: $n=5$. (e) TNFa levels secreted by primary cultured microglia are reduced after incubation with NSC-conditioned medium (CM) vs. control medium treatment (CTR). ${ }^{*} P<0.01$. (f) Representative pictures showing Iba1 staining of primary microglia cultured in CTR or CM. Scale bar $100 \mu \mathrm{m}$. (g) Quantification of Iba1-positive signal per cell in CM vs. CTR condition. ${ }^{*} P<0.01$. e+g: CTR vs. CM two-tailed, unpaired Student's t-test.

Besides possible integration of the NSCs to replace damaged tissue, the beneficial effects we observe in HI mice after NSC treatment can also be explained by indirect secretory effects of the grafted NSCs. The inhibition of microglial activation by the NSCs secretome as shown in our study indicates that NSCs secrete anti-inflammatory factors that dampen the inflammation at the injury site thereby preventing aggravation of HI brain damage.

In addition, NSCs might as well secrete growth factors that can boost endogenous repair and produce certain chemokines thereby attracting endogenous progenitor cells to the lesion to boost endogenous repair. This hypothesis on secretory effects of stem cells is supported by previous work of our group on transplantation of mesenchymal stem cells (MSCs) which induced repair of HI brain injury by supporting proliferation and differentiation of endogenous stem cells into new neurons and oligodendrocytes without actual integration of the MSCs into the brain $(16,27)$. In line with this finding, it would be interesting to test whether the secretome of NSC as such could have a regenerative effect on neonatal HI brain injury. For example, it would be relevant to investigate the effect of transplantation of NSC-derived exosomes, as these vesicles have been already proposed as a new tool to repair brain damage $(28,29)$. Most recently, Ophelders et al., showed that MSC-derived exosomes were beneficial in an ovine model of HI (30). 
When comparing our previous work on MSC transplantation to the current work on NSC transplantation there are a few pros and cons to either of the transplanted cell types. Both types of stem cells seem to have a potent regenerative capacity on HI brain damage, although intracranial injection of similar amounts of MSCs locally in the damaged hippocampus seem to have less effect on improvement of motor behavior than NSC transplantation in the current study $(31,32)$. Transplanted NSCs themselves seem to integrate into the brain and form new neuronal precursors, which could, at least partially, contribute to repair of damaged tissue. Our previous studies using MSC therapy have shown that MSCs are short-lived after transplantation in the brain and do not integrate $(27,31)$. Our previous work together with the current study show that transplantation of both stem cell types reduces the neuro-inflammatory response probably due to secretory effects of the stem cells and that both types of stem cells can boost endogenous repair (27). A possible drawback of the current study with NSCs is that we used an invasive route of administration which makes it hard to translate to the clinical setting. Administration through other routes (e.g., intravenous or intranasal) might be more attractive if proven as efficient. Our current data show that NSCs are capable of migrating from the contralateral hippocampus towards the damaged ipsilateral site. This observation illustrates the great potential of applying NSCs at sites more distant from the lesion, e.g., via a noninvasive route via the nose, as we have shown to be effective for MSCs. Studying the potential of intranasal NSC transplantation will be subject of our future research. Furthermore, it would be of great interest to study the effect of delayed NSC treatment in combination with hypothermia, the latter being the only efficacious treatment in the clinic at present.

In addition, a possible disadvantage of NSCs over MSCs is the fact that NSCs are harder to isolate and expand when compared to MSCs. MSCs can relatively easy be isolated from bone marrow or adipose tissue of the patient (33), while NSCs have to be isolated from postmortem tissues or derived from induced pluripotent stem cells (34). We show here as proof of principle the use and efficacy of embryonic allogenic NSCs. For human application, the use of embryos as source of transplantable cells is ethically impeded; therefore, future efforts should focus on different sources of NSCs (e.g., the above mentioned induced pluripotent cell-derived NSCs). Further studies in that direction are required in order to bring NSC transplantation closer toward a clinical application for neonatal patients suffering from HI brain injury.

\section{METHODS}

NSCS

NSCs derived from embryonic day 14.5 CD-1 mice were purchased at R\&D Systems (Minneapolis, MN) and cultured as neurospheres in DMEM:F12 medium (Thermo Scientific, Waltham, MA) supplemented with $50 \times$ B-27 (Thermo scientific) plus $20 \mathrm{ng} / \mathrm{ml}$ EGF and $20 \mathrm{ng} / \mathrm{ml}$ bFGF (both Peprotech, Rocky Hill, NJ) and antibiotics (Thermo Scientific). Prior to administration, neurospheres were dissociated into single cells and resuspended in PBS. For in vivo tracing, NSCs were labeled with PKH26 Red fluorescent cell linker kit according to manufacturer's instructions (Sigma-Aldrich, St. Louis, MO).
NSCs were characterized nestin-positive at the time of transplantation indicating progenitor state.

NSC-conditioned medium (CM) was generated by growing neurospheres for $72 \mathrm{~h}$. The medium was then filtered with a $0.2 \mu \mathrm{m}$ sterile filter to remove the NSCs.

\section{Animals}

All experiments were performed in accordance to international guidelines and approved by Experimental Animal Committee Utrecht (DEC, University Utrecht, Utrecht, Netherlands). Briefly, 9-d-old (P9) C57BL/6J mice underwent HI by permanent right carotid artery occlusion under isoflurane anesthesia (4\% induction, $1.5 \%$ maintenance) followed by $45 \mathrm{~min}$ exposure to $10 \%$ oxygen (35). This procedure results in unilateral damage to hippocampus, neocortex, and striatum (36). Sham-operated control animals underwent anesthesia and incision only. In total 43 pups of both genders from 8 different litters were randomly distributed amongst every experimental group. No significant gender differences were identified at any of the measured parameters. All analyses were performed in a blinded set-up.

At day 10 after HI, $1 \times 10^{5}$ NSCs resuspended in 2 il PBS or vehicle $(2 \mu \mathrm{l}$ PBS $)$ were injected under isoflurane anesthesia at $2 \mathrm{~mm}$ caudal to bregma, $1,5 \mathrm{~mm}$ right from midline, and $4 \mathrm{~mm}$ below dural surface in order to reach the ipsilateral hippocampus. For the migration experiment, similar volume and numbers of NSCs were injected into the contralateral hippocampus (similar coordinates but left from midline).

Animals were sacrificed at day 13 ( 3 d post-treatment; NSC $n=6)$, day 15 ( 5 d post-treatment; SHAM $n=3$, VEH $n=3$, NSC $n=5$ ) or day 56 (46 d post-treatment; SHAM $n=9$, VEH $n=9$, NSC $n=9$ ) after $\mathrm{HI}$ by overdose pentobarbital. Animals were transcardially perfused with PBS followed by $4 \%$ formaldehyde. Brains were collected and postfixed in $4 \%$ formaldehyde.

\section{Cylinder Rearing Test}

The cylinder rearing test (CRT) was used to assess forelimb use asymmetry as described before $(36,37)$. Animals were tested in the CRT at $10 \mathrm{~d}$ (prior to NSC treatment), $28 \mathrm{~d}$, and $56 \mathrm{~d}$ after HI. In short, mice were individually placed in a Plexiglas cylinder and observed for $3 \mathrm{~min}$. The forepaw that was used to contact the cylinder wall during a weight-bearing full rear was scored as left ( $\mathrm{L}$; impaired), right (R; nonimpaired), or both. Nonimpaired $(\mathrm{R})$ paw preference was calculated as follows: $((\mathrm{R}-\mathrm{L}) /(\mathrm{L}+\mathrm{R}+$ both $)) \times 100 \%(16,36)$. Inclusion criterion was a minimum of 10 weight-bearing movements in $3 \mathrm{~min}$. The following animals were excluded as they did not meet these inclusion criteria: 1 SHAM, 2 VEH at $10 \mathrm{~d} ; 2$ NSC at $28 \mathrm{~d}, 3 \mathrm{VEH}$ at $56 \mathrm{~d}$. The test was performed by a trained observer blinded to treatment.

\section{Histology and Immunohistochemistry}

Brains collected at $56 \mathrm{~d}$ post-HI were embedded in paraffin. Coronal sections $(8 \mu \mathrm{m})$ were cut at hippocampal level $(-1.8 \mathrm{~mm}$ from bregma) and stained with hematoxylin-eosin (HE) (Klinipath, Duiven, the Netherlands). HE staining was used to assess ipsilateral hemispheric area loss. Other coronal sections were immunohistochemically stained with mouse anti-microtubule-associated protein 2 (MAP2) (Sigma-Aldrich, 1:1,000), mouse anti-myelin basic protein (MBP) (Sternberger Monoclonals, Lutherville, MD, 1:1,600), or mouse antisynaptophysin (Abcam, Cambridge, UK, 1:20) followed by biotinylated horse anti-mouse antibody (Vector Laboratories, Burliname, $\mathrm{CA}$ ) and revealed using Vectastain $\mathrm{ABC}$ kit (Vector Laboratories). Stainings for MAP2 and MBP were used to assess neuronal damage and white matter damage respectively. Synaptophysin, which stains for small synaptic vesicles was used to assess neuronal integrity and axonal sprouting/synaptogenesis. HE-positive or MAP2-positive areas in the ipsilateral and contralateral hemisphere were outlined manually with image processing tools in Adobe Photoshop. The area of MBP staining in both hemispheres was quantified using Image J software (38). Ipsilateral area loss (HE), MAP2 loss, or MBP loss was calculated as (1-(ipsilateral positive area/contralateral positive area)) ${ }^{\star} 100 \%$. For synaptophysin, photographs were taken in the CA3 and DG areas of the hippocampus with a Zeiss Axio Lab A1 microscope and Icc5 camera (Carl Zeiss, Oberkochen, Germany). 


\section{Primary Microglia Experiments}

Primary mixed glial cell cultures were obtained of P0-2 Sprague Dawley rat brains as described by Chen et al.(39). Glial cultures were kept for at least $10 \mathrm{~d}$ in vitro before microglia isolation. Mixed glial cell cultures were shaken on an orbital shaker for $1 \mathrm{~h}$ at $200 \mathrm{rpm}$ to detach microglia. Microglia were plated at 300,000 cells per well onto poly-L-ornithine-coated 24-well plates in DMEM with $10 \%$ FCS and antibiotics. After $24 \mathrm{~h}, 500 \mu \mathrm{l}$ of NSC-conditioned medium (CM) or control medium (nonconditioned NSC growth medium; CTR) were added to the cultures for $24 \mathrm{~h}$. Supernatants were collected and analyzed for TNF $\alpha$ production by using ELISA according to manufacturer's instructions (Ucytech, Utrecht, The Netherlands).

\section{Immunofluorescence}

Brains collected at 13 and $15 \mathrm{~d}$ post-HI were cryoprotected in grading 30-50\% sucrose and embedded in OCT freezing medium (TissueTec, Sajura Zoeterwoude, The Netherlands). Coronal cryosections (10 $\mu \mathrm{m})$ were incubated with rabbit-anti-Ibal (Wako Chemicals, Osaka, Japan, 1:500), mouse anti-GFAP (Acris antibodies, San Diego, CA, 1:100), goat anti-DCX (Santa Cruz Biotechnology, Dallas, TX, 1:100), or mouse anti-Nestin (BD Biosciences, Breda, The Netherlands, 1:100) followed by incubation with secondary antibodies donkey anti-rabbit AF680, donkey anti-mouse AF488, donkey anti-goat AF680 (all Thermo Scientific, 1:250). Nuclei were counterstained with 4',6-diamidino-2-phenylindole (DAPI) (Sigma Aldrich, 1:2,000) and sections were mounted with FluorSave reagent (Merck Millipore, Darmstadt, Germany). Fluorescent confocal images were captured with an AxioCam MRm (Carl Zeiss, Sliedrecht, The Netherlands) on a LSM 700 confocal microscope (Carl Zeiss). Iba1 and GFAP intensity was quantified using Image J software by measuring the staining intensity in five different fields of the hippocampus.

For staining of the microglia cultures, cells were fixed in $4 \%$ formaldehyde and stained with rabbit-anti-Iba1 (1:500), followed by incubation with donkey-anti-rabbit AlexaFluor-555 (Thermo Scientifics). Nuclei were counterstained with DAPI. Images were acquired using the EVOS FL Cell Imaging System (Thermo Scientifics). The expression level of Ibal per cell was determined using Image J software.

\section{Statistics}

Data are expressed as mean \pm SEM and regarded statistically significant if $P<0.05$. Data were analyzed using one- or two-way ANOVA with Fisher's least significant difference (LSD) post-test. For the conditioned medium experiments, two-tailed, unpaired Student's $t$-test was used. Outliers were determined with the Grubbs' test.

\section{ACKNOWLEDGMENTS}

L.B. and C.H.N. conceived and performed the experiments, analyzed the data and wrote the manuscript. P.J.C. and C.J.H. helped in designing the experiment and revised the manuscript. The authors would like to thank Erik van Tilborg, Elke Kooijman, Caren van Kammen, and Sabrina Renes for their excellent technical assistance.

\section{STATEMENT OF FINANCIAL SUPPORT}

No financial assistance was received in support of the study.

Disclosure: The authors declare no conflict of interest.

\section{REFERENCES}

1. Reynolds BA, Weiss S. Generation of neurons and astrocytes from isolated cells of the adult mammalian central nervous system. Science 1992;255:1707-10.

2. Palmer TD, Willhoite AR, Gage FH. Vascular niche for adult hippocampal neurogenesis. J Comp Neurol 2000;425:479-94.

3. Giachino $\mathrm{C}$, Basak $\mathrm{O}$, Taylor V. Isolation and manipulation of mammalian neural stem cells in vitro. Methods Mol Biol 2009;482:143-58.

4. Martino G, Pluchino S. The therapeutic potential of neural stem cells. Nat Rev Neurosci 2006;7:395-406.

5. Blurton-Jones M, Kitazawa M, Martinez-Coria $\mathrm{H}$, et al. Neural stem cells improve cognition via BDNF in a transgenic model of Alzheimer disease. Proc Natl Acad Sci USA 2009;106:13594-9.
6. Pluchino S, Quattrini A, Brambilla E, et al. Injection of adult neurospheres induces recovery in a chronic model of multiple sclerosis. Nature 2003;422:688-94.

7. MendonçaLS, Nóbrega C, HiraiH,Kaspar BK, Pereira de AlmeidaL. Transplantation of cerebellar neural stem cells improves motor coordination and neuropathology in Machado-Joseph disease mice. Brain 2015;138(Pt 2):320-35.

8. Titomanlio L, Bouslama M, Le Verche V, et al. Implanted neurospherederived precursors promote recovery after neonatal excitotoxic brain injury. Stem Cells Dev 2011;20:865-79.

9. de Haan M, Wyatt JS, Roth S, Vargha-Khadem F, Gadian D, Mishkin M. Brain and cognitive-behavioural development after asphyxia at term birth. Dev Sci 2006;9:350-8.

10. Ferriero DM. Neonatal brain injury. N Engl J Med 2004;351:1985-95.

11. Buono KD, Goodus MT, Guardia Clausi M, Jiang Y, Loporchio D, Levison SW. Mechanisms of mouse neural precursor expansion after neonatal hypoxia-ischemia. J Neurosci 2015;35:8855-65.

12. Felling RJ, Snyder MJ, Romanko MJ, et al. Neural stem/progenitor cells participate in the regenerative response to perinatal hypoxia/ischemia. J Neurosci 2006;26:4359-69.

13. Brazel CY, Rosti RT 3rd, Boyce S, Rothstein RP, Levison SW. Perinatal hypoxia/ischemia damages and depletes progenitors from the mouse subventricular zone. Dev Neurosci 2004;26:266-74.

14. Daadi MM, Davis AS, Arac A, et al. Human neural stem cell grafts modify microglial response and enhance axonal sprouting in neonatal hypoxicischemic brain injury. Stroke 2010;41:516-23.

15. Donega V, van Velthoven CT, Nijboer CH, et al. Intranasal mesenchymal stem cell treatment for neonatal brain damage: long-term cognitive and sensorimotor improvement. PLoS One 2013;8:e51253.

16. van Velthoven CT, Kavelaars A, van Bel F, Heijnen CJ. Repeated mesenchymal stem cell treatment after neonatal hypoxia-ischemia has distinct effects on formation and maturation of new neurons and oligodendrocytes leading to restoration of damage, corticospinal motor tract activity, and sensorimotor function. J Neurosci 2010;30:9603-11.

17. Gluckman PD, Wyatt JS, Azzopardi D, et al. Selective head cooling with mild systemic hypothermia after neonatal encephalopathy: multicentre randomised trial. Lancet 2005;365:663-70.

18. Azzopardi DV, Strohm B, Edwards AD, et al.; TOBY Study Group. Moderate hypothermia to treat perinatal asphyxial encephalopathy. N Engl J Med 2009;361:1349-58.

19. Ashwal S, Ghosh N, Turenius CI, et al. Reparative effects of neural stem cells in neonatal rats with hypoxic-ischemic injury are not influenced by host sex. Pediatr Res 2014;75:603-11.

20. Rosenblum S, Smith TN, Wang N, et al. BDNF Pretreatment of Human Embryonic-Derived Neural Stem Cells Improves Cell Survival and Functional Recovery After Transplantation in Hypoxic-Ischemic Stroke. Cell Transplant 2015;24:2449-61.

21. Ito D, Tanaka K, Suzuki S, Dembo T, Fukuuchi Y. Enhanced expression of Iba1, ionized calcium-binding adapter molecule 1, after transient focal cerebral ischemia in rat brain. Stroke 2001;32:1208-15.

22. Kuno R, Wang J, Kawanokuchi J, Takeuchi H, Mizuno T, Suzumura A. Autocrine activation of microglia by tumor necrosis factor-alpha. J Neuroimmunol 2005;162:89-96.

23. Bonestroo HJ, Heijnen CJ, Groenendaal F, van Bel F, Nijboer CH. Development of cerebral gray and white matter injury and cerebral inflammation over time after inflammatory perinatal asphyxia. Dev Neurosci 2015;37:78-94.

24. Rosenblum S, Wang N, Smith TN, et al. Timing of intra-arterial neural stem cell transplantation after hypoxia-ischemia influences cell engraftment, survival, and differentiation. Stroke 2012;43:1624-31.

25. Park KI, Teng YD, Snyder EY. The injured brain interacts reciprocally with neural stem cells supported by scaffolds to reconstitute lost tissue. Nat Biotechnol 2002;20:1111-7.

26. Imitola J, Raddassi K, Park KI, et al. Directed migration of neural stem cells to sites of CNS injury by the stromal cell-derived factor 1alpha/CXC chemokine receptor 4 pathway. Proc Natl Acad Sci USA 2004;101:18117-22.

27. Donega V, Nijboer CH, van Tilborg G, Dijkhuizen RM, Kavelaars A, Heijnen CJ. Intranasally administered mesenchymal stem cells promote a regenerative niche for repair of neonatal ischemic brain injury. Exp Neurol 2014;261:53-64. 
28. Braccioli L, van Velthoven C, Heijnen CJ. Exosomes: a new weapon to treat the central nervous system. Mol Neurobiol 2014;49:113-9.

29. Xin H, Li Y, Cui Y, Yang JJ, Zhang ZG, Chopp M. Systemic administration of exosomes released from mesenchymal stromal cells promote functional recovery and neurovascular plasticity after stroke in rats. J Cereb Blood Flow Metab 2013;33:1711-5.

30. Ophelders DR, Wolfs TG, Jellema RK, et al. Mesenchymal Stromal CellDerived Extracellular Vesicles Protect the Fetal Brain After HypoxiaIschemia. Stem Cells Transl Med 2016;5:754-63.

31. van Velthoven CT, Kavelaars A, van Bel F, Heijnen CJ. Mesenchymal stem cell transplantation changes the gene expression profile of the neonatal ischemic brain. Brain Behav Immun 2011;25:1342-8.

32. van Velthoven CT, van de Looij Y, Kavelaars A, et al. Mesenchymal stem cells restore cortical rewiring after neonatal ischemia in mice. Ann Neurol 2012;71:785-96.

33. Hass R, Kasper C, Böhm S, Jacobs R. Different populations and sources of human mesenchymal stem cells (MSC): A comparison of adult and neonatal tissue-derived MSC. Cell Commun Signal 2011;9:12.
34. Liu J. Induced pluripotent stem cell-derived neural stem cells: new hope for stroke? Stem Cell Res Ther 2013;4:115.

35. Nijboer CH, Kavelaars A, Vroon A, Groenendaal F, van Bel F, Heijnen CJ. Low endogenous G-protein-coupled receptor kinase 2 sensitizes the immature brain to hypoxia-ischemia-induced gray and white matter damage. J Neurosci 2008;28:3324-32.

36. van der Kooij MA, Ohl F, Arndt SS, Kavelaars A, van Bel F, Heijnen CJ. Mild neonatal hypoxia-ischemia induces long-term motor- and cognitive impairments in mice. Brain Behav Immun 2010;24:850-6.

37. Schallert T, Fleming SM, Leasure JL, Tillerson JL, Bland ST. CNS plasticity and assessment of forelimb sensorimotor outcome in unilateral rat models of stroke, cortical ablation, parkinsonism and spinal cord injury. Neuropharmacology 2000;39:777-87.

38. Schneider CA, Rasband WS, Eliceiri KW. NIH Image to ImageJ: 25 years of image analysis. Nat Methods 2012;9:671-5.

39. Chen Y, Balasubramaniyan V, Peng J, et al. Isolation and culture of rat and mouse oligodendrocyte precursor cells. Nat Protoc 2007;2:1044-51. 